\title{
NodeMCU based Electrical Appliances Control using Internet of Things and Network of Things
}

\author{
Ravin N. Sardhara \\ Department of Electronics \& Communication \\ Engineering \\ V.V.P. Engineering College \\ Rajkot, India
}

\author{
Nirmal R. Bhalani \\ Department of Electronics \& Communication \\ Engineering \\ V.V.P. Engineering College \\ Rajkot, India
}

\begin{abstract}
Network of Things (NoT) and Internet of Things (IoT) is a shared network of objects (things) which can interact with each other while NoT is utilized while unavailability of internet connection and IoT is utilized while providing the cyber world connection. These both the protocols widely play a paramount role in industrial automation and home automation individually. The practical goal of this article has been to engender a frugal, utilize amicable and captivating system for home automation system by the utilization of these both the systems. The web application or android mobile is fundamentally used to send the commands to the Node-MCU to control all the habitation appliances. The main feature of this system is to switch on/off predicated on android app or web application with speed control of fan predicated on temperature variation, vicissitude in intensity of tube light or lamp predicated on light intensity of sunlight. This proposed system has withal another feature is to get the status of home appliances from android mobile phone or web application.
\end{abstract}

\section{Keywords}

(Internet of Things), NoT (Network of Things), Node MCU, ESP 8266, Home Automation.

\section{INTRODUCTION}

In the field of electronics and communication, people are generally wanted to automate sundry industrial and home appliances. By denotes of home automation, mechanical switches should be superseded by sundry technology predicated relay controlled board. The desideratum of home automation brought many revolutions in the subsisting and preceding technologies. These can be utilized as a supersession of the subsisting switches in home which may engender sparks and additionally results in fire accidents in some situations.

Sundry wireless technologies like IR, RFID, ZIGBEE, Bluetooth, GSM and Wi-Fi are mainly utilized with the microcontroller for the automation purport. Each technology has its own unique technical designations and applications. From all of the above technologies, considering the advantages of Wi-Fi with internet connectivity, an advanced automation system can be developed to control the appliances in the house. In this paper, we described about controlling all the habitation appliances utilizing android application and web application. All the electrical appliances are fundamentally controlled by Node MCU with android application and web application. Basically Node MCU consists of micro controller and in built Wi-Fi module. Wi-Fi Module is basically used to receive the commands from astute phone in case of NoT (Network of Things) and from android application and Web Application in case of IoT (Internet of Things) and further processed by Node MCU Board itself. In this design android application has developed by MIT app inventor and controlling application was developed utilizing Arduino IDE. This proposed system can monitor and control all the abode appliances.

\section{LITERATURE REVIEW}

It has been found that home automation systems were developed by using various different technologies. If we consider various technologies, when using a home automation system based on GSM, we need to send a message / make a call to control the appliances. It has more delay time and a complex system. In this system, it is found that there is a serious issue of getting proper mobile network. Once this network connection gets cut, all over system gets stuck and no further actions can be executed by using this system. In another way, by using the Bluetooth based home automation system, we can also control all the appliances but the main disadvantage is the range as range of Bluetooth module HC 05 is very limited.

So that specifically it can be used in small home area and it can't extended in industrial area. Some developers have designed home automation using Wi-Fi shield with Arduino board using micro controller but in those designs they implemented only ON / OFF functionalities. In our design we implement the ON / OFF functionality together with the voltage variations and all the appliances are controlled through the use of Android applications and several sensors. Here in this proposed system, sensors like LM 35, PIR and LDR are basically used to automate the home appliances like Lamp, speed of Fan, AC etc.

\section{DESIGN AND IMPLEMENTATION}

The following figure [1] shows the proposed home automation system or electrical appliances control system which can be controlled by web application and android application. In this system we have designed dedicated Web Application and android application for home automation. Adding to this, Single android application is designed in such a way that, Network of Things (NoT) and Internet of Things (IoT) are included so that within single application, user can control all home appliances.

The system that we are using Node MCU, PIR sensor, temperature sensor LM 35 and LDR. The control signal can be received by local Wi Fi hotspot or web application/android application and it is used to drive the relay board.

By this way, we can control (on/off) the various appliances. This proposed system can be extended up to industry automation also. 

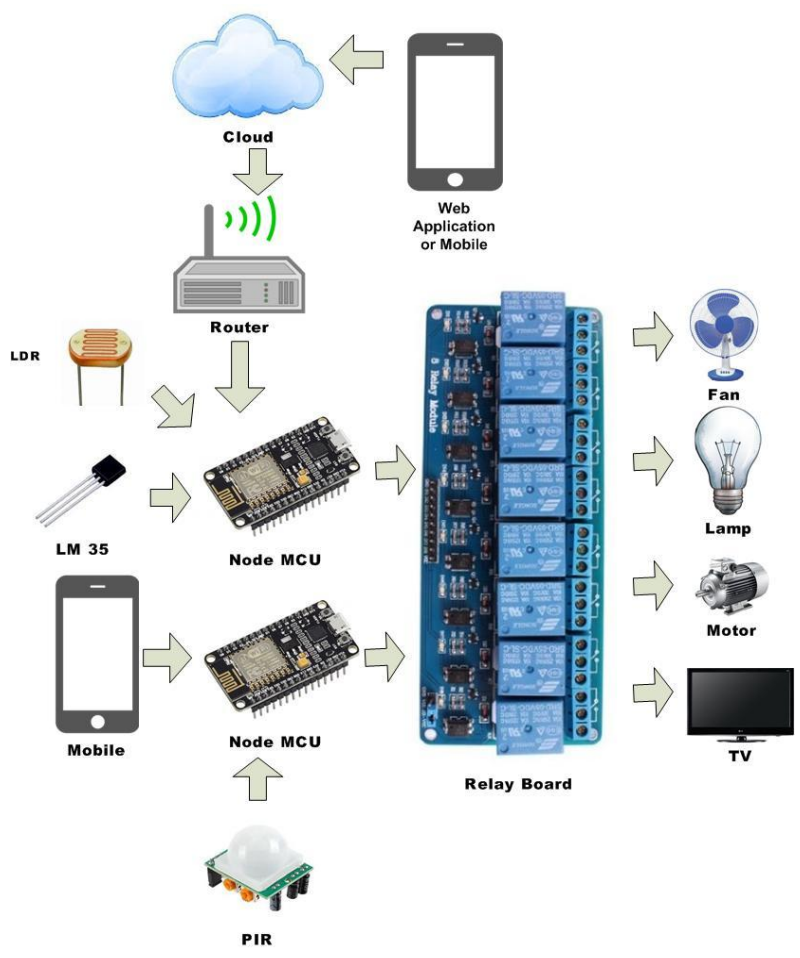

Fig 1: IoT and NoT based home automation System

\subsection{LDR}

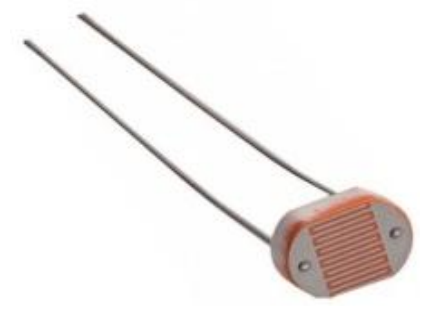

Fig 2: LDR (Light Dependant resistor)

An LDR is a sensor that changes its resistance across two terminals based on changes in intensity of light thrown on it. As the light intensity increases, the conductivity across terminal changes that leads to decrease the resistance. This mechanism allows it to be used as light sensing elements. In this proposed system LDR is basically used as a light sensing elements to change the intensity of Tube light and lamp

\subsection{Temperature Sensor (LM 35)}

LM35 is used to detect temperature sensors. The advantages of temperature levels in our home is the LM 35 sensor has its low cost and high sensitivity between $+2^{\prime} \mathrm{C}$ and $+250^{\prime} \mathrm{C}$ and lower power only $+5 \mathrm{~V}$ DC usage.

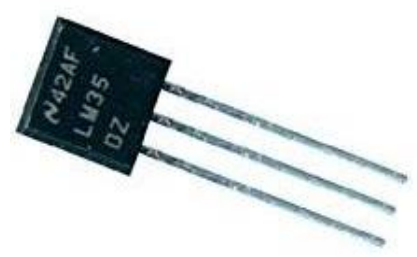

Fig 3: Temperature sensor (LM35)
As room temperature increases, output of LM 35 gets increased and it can be detected using Node MCU. With the use of TRIAC, we can control the speed of fan

\subsection{PIR sensor}

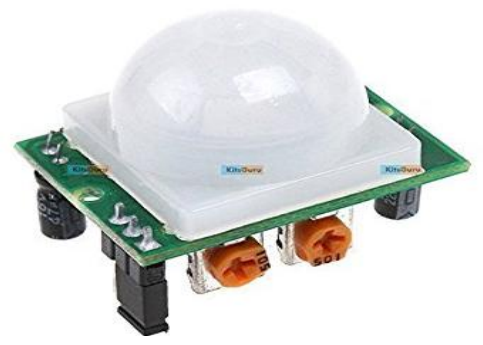

Fig 4: PIR Sensor

A passive infrared PIR sensor is an electronic transducer that measures infrared light radiating from objects in its field of view. They are most often used in PIR-based motion detectors. This module is basically used to automatically switch on the light and fan based output of PIR sensor. Here in this proposed project, automatically light can be controlled using output of PIR sensor that is actually connected with Node MCU.

\subsection{ESP 8266}

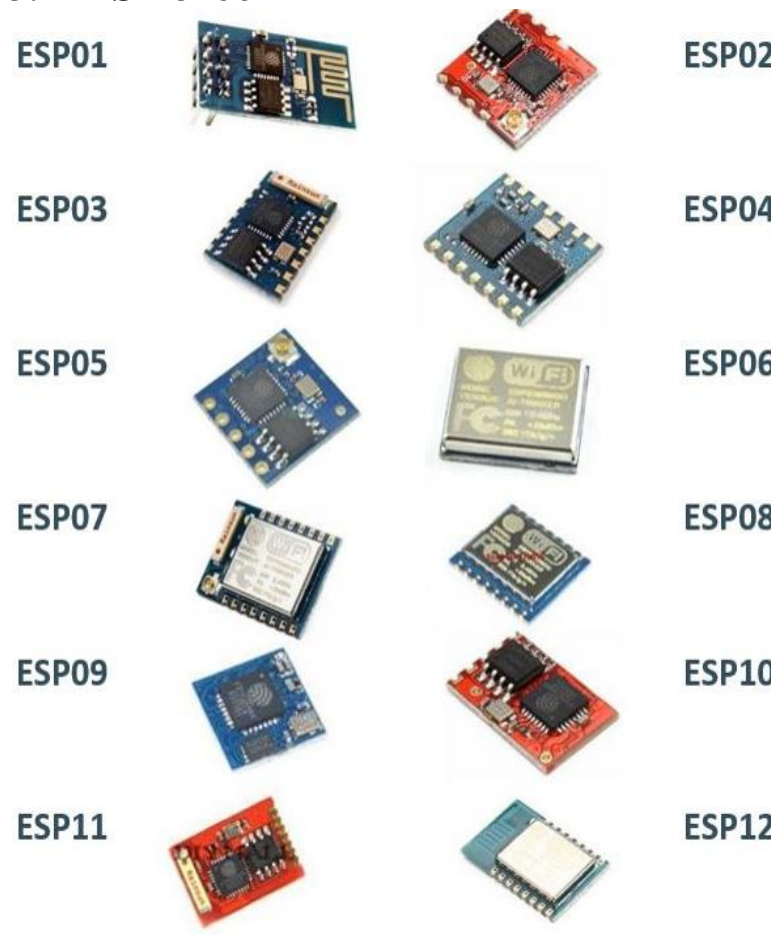

Fig 5: Various versions of ESP module

ESP8266 WiFi Module is a self contained SoC with integrated TCP/IP protocol stack. It is capable of either hosting an application or offloading all WiFi networking functions from another application processor. ESP8266 comes packed with a powerful controller and no external controller interfacing is required. Here in the above figure, various versions of ESP modules are shown. ESPRESSIF provides various version of ESP modules of which ESP12 module is very popular. It is also low cost chip with small PCB area and power efficiency. 


\subsection{NodeMCU}

NodeMCU is an open source IoT platform which includes firmware which runs on the ESP8266 Wi-Fi SoC from Espressif Systems and hardware which is based on the ESP-

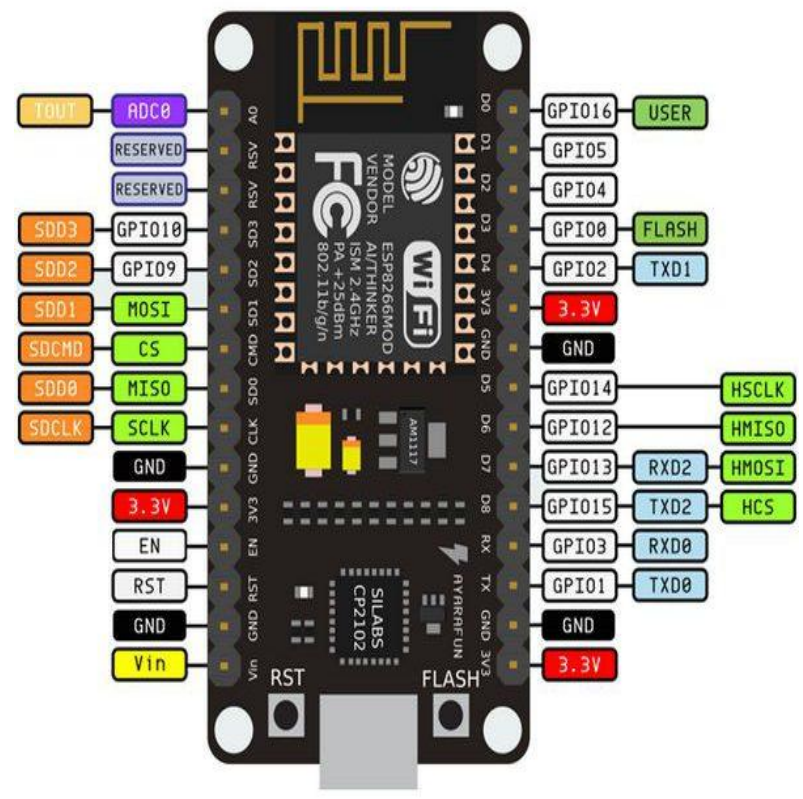

Fig 6: Node MCU Pin diagram

12 module. The term "NodeMCU" by default refers to the firmware rather than the dev kits. The firmware uses the Lua scripting language.

\section{RESULTS AND DISCUSSIONS}

Results of Web Application and Android Application is as given below

\subsection{Web Application}

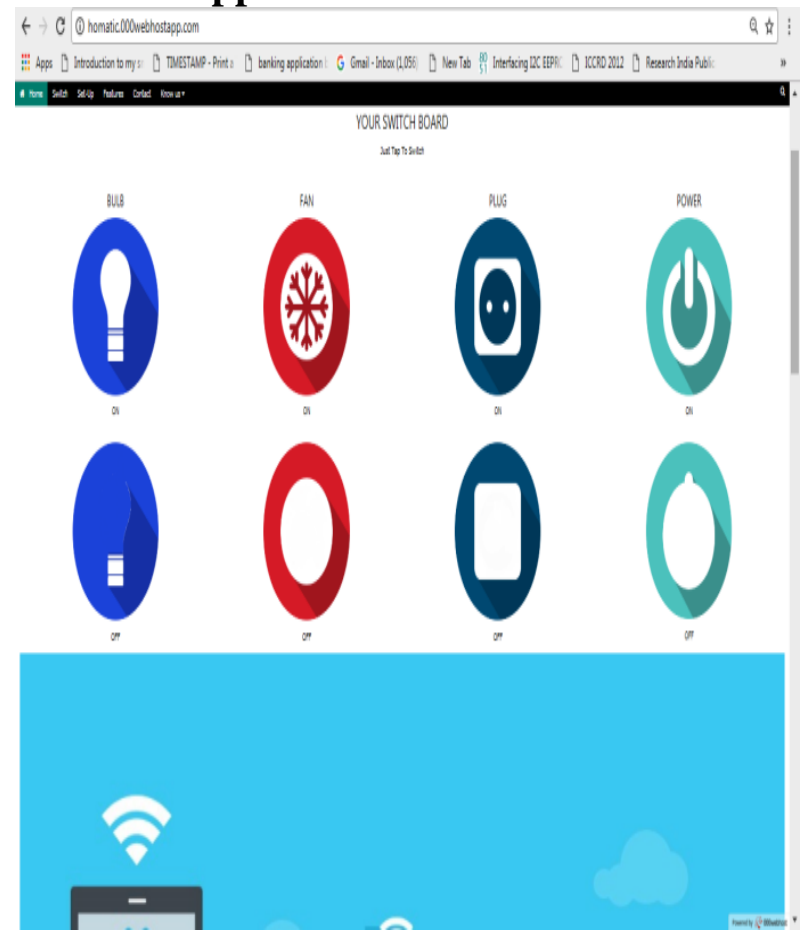

Fig 7: Web Application for home automation
We have designed a web application for the home automation. There is various free web hosting sites through which we can design web application and once the all over system gets finalized, we can design web application on personal web host domain.

\subsection{Android Application}

We have designed an android application for sending control signals through local Wi Fi network and over internet connection.
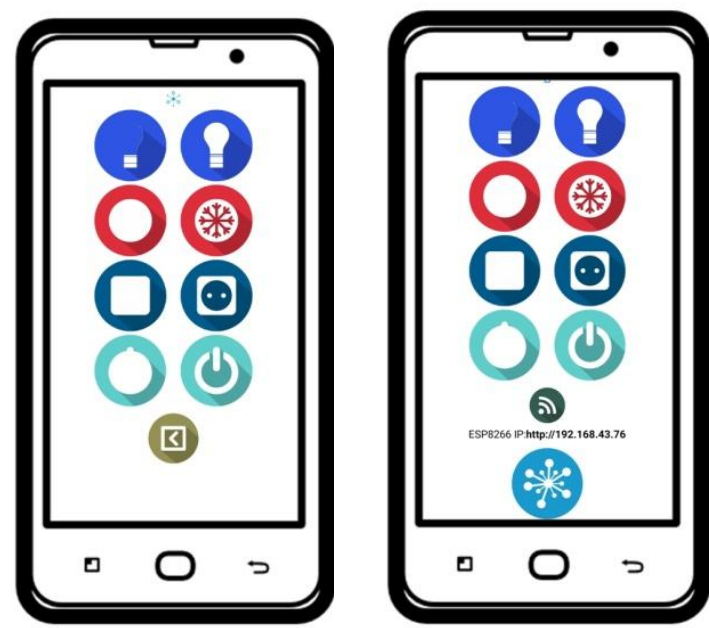

Fig 8: screenshot of Android application (IoT \& NoT)

In NoT (network of Things), after establishing connection we can control the home appliances using android application as shown in above figure. For that we need to provide IP address of node MCU for establishment of connection as there is no requirement of internet connectivity in Node MCU. So in this proposed system, one Node MCU has to configure as Host to provide wifi connectivity.

In IoT (Internet of Things), another Node MCU is configured as a Node. This node MCU is connected to router for fetching internet connectivity. So that though this internet connectivity, node MCU can fetch control signal though android application or web application. Here we are controlling four appliances like Fan, Motor, Lamp and TV. As per user requirement we can connect more number of devices.

In this system, we can control various appliances like lamp and speed of fan based on variation of output of sensors like LDR and LM35 respectively. Additionally with the use of PIR sensor, we can automate the switch on/switch off the light.

We can also control the various appliances using web application. Same website is linked up to android application so with the use of network of things (NoT) and Internet of Things (IoT), we can control the various appliances through android application and web application.

\section{ACKNOWLEDGMENT}

We are gratified to the Department of Electronics and Communication Engineering, VVP Engineering College and students and colleagues for the support rendered to us in carrying out this work. 


\section{REFERENCES}

[1] Zhai Y., Cheng X., (2011), "Design of Smart Home Remote Monitoring System Based on Embedded System", Control and Industrial Engineering, IEEE 2nd International Conference, pp.41-44.

[2] Gurek A., Gur C., Gurakin C., Akdeniz M., Metin S. K., Korkmaz I., (2013), "An Android Based Home Automation System", High Capacity Optical Networks and Enabling Technologies, IEEE 10th International Conference on, pp.121- 125.

[3] Yüksekkaya B., Kayalar A. A., Tosun M. B., Özcan M. K., Alkar A. Z., (2006), "A GSM, Internet and Speech Controlled Wireless Interactive Home Automation System", Consumer Electronics, IEEE Transactions on, vol.52, no.3, pp.837-843.
[4] Yamazaki T., (2006), "Beyond the Smart Home", Hybrid Information Technology, IEEE International Conference on, vol.2, pp.350-355.

[5] Sriskanthan N., Tan F., Karande A., (2002), "Bluetooth Based Home Automation", Microprocessors and Microsystems, Elsevier Science B.V. vol.26, no.6, pp.281-289.

[6] EASAMBATTU, Thejaswini; REDDY, P. Ajay Kumar; RAMAIAH, G. N. Kodanda. Controlling Home Appliances through GSM Modem and Internet. International Journal of Electronics Engineering Research, [S.1.], p. 1-7, oct. 2013. ISSN 0975- 6450 